\title{
Molecular Imaging of Prostate Cancer: Choosing the Right Agent
}

\author{
Steven P. Rowe ${ }^{1,2}$, Martin G. Pomper ${ }^{1,2}$, and Michael A. Gorin ${ }^{1,2}$ \\ ${ }^{1}$ Russell H. Morgan Department of Radiology and Radiological Science, Johns Hopkins University School of Medicine, Baltimore, \\ Maryland; and ${ }^{2}$ James Buchanan Brady Urological Institute and Department of Urology, Johns Hopkins University School of \\ Medicine, Baltimore, Maryland
}

See the associated article on page 789 .

he well-recognized limitations of conventional imaging with CT, MRI, and ${ }^{99 m}$ Tc-methylene diphosphonate bone scans have contributed to a revolution in PET imaging of prostate cancer (PCa). A plethora of PET radiotracers has entered preclinical and early clinical development, and in fact, 2 compounds have been approved by the U.S. Food and Drug Administration for PCa imaging ( ${ }^{11} \mathrm{C}$-choline (1) and anti-1-amino-3- ${ }^{18} \mathrm{~F}$-fluorocyclobutane-1-carboxylic acid [ ${ }^{18} \mathrm{~F}$-fluciclovine] (2)). Furthermore, at least 2 different radiotracers targeting prostate-specific membrane antigen (PSMA) are likely to undergo the New Drug Application process at the Food and Drug Administration within the next few years ( ${ }^{68}$ Ga-PSMA-11 (3) and ${ }^{18}$ F-DCFPyL (4)).

However, as these multiple radiotracers become more widely available, it will become necessary for clinicians who treat men with PCa to choose among these agents in an informed manner. That is not a trivial matter, as different radiotracers may have advantages or disadvantages that affect their utility in different clinical scenarios (5), and all radiotracers have pitfalls to interpretation that may be more salient in some patients than in others (6). Understanding these nuances and being able to recommend the appropriate radiotracer in different circumstances will undoubtedly be important foundational knowledge for nuclear imaging specialists.

This precept underlies the importance of the article by Calais et al. that appears in this issue of The Journal of Nuclear Medicine: "Comparison of ${ }^{68} \mathrm{Ga}$-PSMA-11 and ${ }^{18} \mathrm{~F}$-Fluciclovine PET/CT in a Case Series of 10 Patients with Prostate Cancer Recurrence" (7). In this study, the authors retrospectively reviewed the records of 288 patients with recurrent $\mathrm{PCa}$ who participated in a prospective study examining the use of ${ }^{68} \mathrm{Ga}$-PSMA-11 PET/CT for disease localization. Ten patients were identified who had also undergone imaging with ${ }^{18} \mathrm{~F}$-fluciclovine PET/CT, at a median of 2.3 mo before study enrollment. The median serum prostate-specific

Received Dec. 26, 2017; revision accepted Dec. 30, 2017.

For correspondence or reprints contact: Steven P. Rowe, Russell H. Morgan Department of Radiology and Radiological Science, Johns Hopkins University School of Medicine, 601 N. Caroline St., Baltimore, MD 21287.

E-mail: srowe8@jhmi.edu

Guest Editor: Michael Graham, University of lowa

Published online Jan. 25, 2018.

COPYRIGHT @ 2018 by the Society of Nuclear Medicine and Molecular Imaging.

DOI: 10.2967/jnumed.117.206318 antigen level of these patients was quite low $(1.0 \mathrm{ng} / \mathrm{mL}$ at the time of ${ }^{18} \mathrm{~F}$-fluciclovine imaging and $1.1 \mathrm{ng} / \mathrm{mL}$ at the time of ${ }^{68}$ Ga-PSMA-11 imaging), thus testing the limits of sensitivity for these 2 radiotracers.

The authors observed starkly different detection efficiencies with the 2 radiotracers. More specifically, ${ }^{18} \mathrm{~F}$-fluciclovine was able to identify putative sites of disease in 2 of 10 patients (20\%), whereas ${ }^{68}$ Ga-PSMA-11-avid foci were seen in 7 of 10 patients (70\%). Of the 8 patients with negative ${ }^{18} \mathrm{~F}$-fluciclovine results, $5(63 \%)$ had suggestive findings with ${ }^{68} \mathrm{Ga}$-PSMA-11 PET/CT. In both of the patients with positive ${ }^{18} \mathrm{~F}$-fluciclovine $\mathrm{PET} / \mathrm{CT}$ results, additional sites of suspected disease were seen with ${ }^{68} \mathrm{Ga}-\mathrm{PSMA}-11$. As noted by the authors, the markedly higher sensitivity of the PSMA-targeted agent led to changes in clinical decision making.

Calais et al. acknowledge that the inherent shortcomings of their small retrospective study limit the conclusions that can be drawn regarding the performance of these 2 radiotracers. The authors' findings, however, are in keeping with the available literature, which would have predicted higher sensitivity for PSMA-targeted agents at low prostate-specific antigen levels $(8-10)$. Indeed, prospective trials are needed in which patients are imaged with both ${ }^{18}$ F-fluciclovine and a PSMA-targeted radiotracer within a short interval (1-7 d). Additionally, these studies should aim to compare these radiotracers across a range of clinical contexts $(11,12)$, including the staging of men presenting with newly diagnosed $\mathrm{PCa}$ who are at risk for harboring occult metastatic disease, as well as in the setting of castration resistance among patients being considered for endoradiotherapy with PSMA-targeted therapeutic agents $(13,14)$. This latter clinical context is of particular importance because PSMA expression is known to decrease with neuroendocrine differentiation $(15,16)$, and therefore imaging with ${ }^{18} \mathrm{~F}$-fluciclovine or other agents in combination with a PSMAtargeted compound may help identify patients who are poor candidates for endoradiotherapy.

The article by Calais et al. is an important step forward in comparing 2 of the most widely studied PCa radiotracers. It is incumbent on the field of molecular imaging to ensure that additional comparative studies are undertaken to firmly establish the roles of different PCa radiotracers in various clinical settings. Only then can we select the right tool for the job. Without these data, we have nothing more than an embarrassment of riches/radiotracers.

\section{DISCLOSURE}

Martin G. Pomper is a coinventor on a U.S. patent covering ${ }^{18} \mathrm{~F}-$ DCFPyL and as such is entitled to a portion of any licensing fees 
and royalties generated by this technology. This arrangement has been reviewed and approved by the Johns Hopkins University in accordance with its conflict-of-interest policies. Michael A. Gorin has served as a consultant to Progenics Pharmaceuticals, the licensee of ${ }^{18}$ F-DCFPyL. Michael A. Gorin, Martin G. Pomper, and Steven P. Rowe have received research support from Progenics Pharmaceuticals. No other potential conflict of interest relevant to this article was reported.

\section{REFERENCES}

1. Hara T, Kosaka N, Kishi H. PET imaging of prostate cancer using carbon-11choline. J Nucl Med. 1998;39:990-995.

2. Schuster DM, Votaw JR, Nieh PT, et al. Initial experience with the radiotracer anti-1-amino-3- ${ }^{18}$ F-fluorocyclobutane-1-carboxylic acid with PET/CT in prostate carcinoma. J Nucl Med. 2007;48:56-63.

3. Afshar-Oromieh A, Haberkorn U, Eder M, Eisenhut M, Zechmann CM. $\left[{ }^{68} \mathrm{Ga}\right]$ gallium-labelled PSMA ligand as superior PET tracer for the diagnosis of prostate cancer: comparison with ${ }^{18} \mathrm{~F}-\mathrm{FECH}$. Eur J Nucl Med Mol Imaging. 2012;39:1085-1086.

4. Szabo Z, Mena E, Rowe SP, et al. Initial evaluation of $\left[{ }^{18} \mathrm{~F}\right] \mathrm{DCFPyL}$ for prostatespecific membrane antigen (PSMA)-targeted PET imaging of prostate cancer. Mol Imaging Biol. 2015;17:565-574.

5. Gorin MA, Pienta KJ, Pomper MG, Rowe SP. Prostate cancer local recurrence detected with both ${ }^{18} \mathrm{~F}$-fluciclovine and PSMA-targeted ${ }^{18} \mathrm{~F}$-DCFPyL PET/CT. Urology. 2017;107:e9-e10.

6. Sheikhbahaei S, Afshar-Oromieh A, Eiber M, et al. Pearls and pitfalls in clinical interpretation of prostate-specific membrane antigen (PSMA)-targeted PET imaging. Eur J Nucl Med Mol Imaging. 2017;44:2117-2136.
7. Calais J, Fendler WP, Herrmann K, Eiber M, Ceci F. Comparison of ${ }^{68} \mathrm{Ga}$-PSMA-11 and ${ }^{18} \mathrm{~F}$-fluciclovine PET/CT in a case series of 10 patients with prostate cancer recurrence. J Nucl Med. 2018;59:789-794.

8. Bach-Gansmo T, Nanni C, Nieh PT, et al. Multisite experience of the safety, detection rate and diagnostic performance of fluciclovine $\left({ }^{18} \mathrm{~F}\right)$ positron emission tomography/computerized tomography imaging in the staging of biochemically recurrent prostate cancer. J Urol. 2017;197:676-683.

9. Eiber M, Maurer T, Souvatzoglou M, et al. Evaluation of hybrid ${ }^{68} \mathrm{Ga}-\mathrm{PSMA}$ ligand PET/CT in 248 patients with biochemical recurrence after radical prostatectomy. J Nucl Med. 2015;56:668-674.

10. Afshar-Oromieh A, Holland-Letz T, Giesel FL, et al. Diagnostic performance of ${ }^{68}$ Ga-PSMA-11 (HBED-CC) PET/CT in patients with recurrent prostate cancer: evaluation in 1007 patients. Eur J Nucl Med Mol Imaging. 2017;44:1258-1268.

11. Rowe SP, Gorin MA, Allaf ME, et al. PET imaging of prostate-specific membrane antigen in prostate cancer: current state of the art and future challenges. Prostate Cancer Prostatic Dis. 2016;19:223-230.

12. Gorin MA, Rowe SP, Denmeade SR. Clinical applications of molecular imaging in the management of prostate cancer. PET Clin. 2017;12:185-192.

13. Weineisen M, Schottelius M, Simecek J, et al. ${ }^{68} \mathrm{Ga}$ - and ${ }^{177} \mathrm{Lu}$-labeled PSMA I\&T: optimization of a PSMA-targeted theranostic concept and first proof-ofconcept human studies. J Nucl Med. 2015;56:1169-1176.

14. Kratochwil C, Bruchertseifer F, Giesel FL, et al. ${ }^{225}$ Ac-PSMA-617 for PSMAtargeted $\alpha$-radiation therapy of metastatic castration-resistant prostate cancer. J Nucl Med. 2016;57:1941-1944.

15. Chakraborty PS, Tripathi M, Agarwal KK, Kumar R, Vijay MK, Bal C. Metastatic poorly differentiated prostatic carcinoma with neuroendocrine differentiation: negative on ${ }^{68} \mathrm{Ga}$-PSMA PET/CT. Clin Nucl Med. 2015;40:e163-e166.

16. Tosoian JJ, Gorin MA, Rowe SP, et al. Correlation of PSMA-targeted ${ }^{18} \mathrm{~F}$ DCFPyL PET/CT findings with immunohistochemical and genomic data in a patient with metastatic neuroendocrine prostate cancer. Clin Genitourin Cancer. 2017;15:e65-e68. 